\title{
Surface Plasmon Resonance for Human Bone Marrow Cells Imaging
}

\author{
Thomas Wilkop, N. Manivannan ${ }^{\circledR}$, Senior Member, IEEE, W. Balachandran, Fellow, IEEE, \\ and Asim K. Ray
}

\begin{abstract}
Surface plasmon resonance imaging (SPRI) detects the changes in refractive index in close proximity to the surface of a thin metal film as variations in light intensity reflected from the back of the film and thus does not require labeling for visualization of the structures under investigation. While traditionally, the wave vector scanning is performed via angular rotations, the wave vector can also be scanned though tuning of the wavelength. Here we demonstrate that a combination of a non-monochromatic electrically tunable bandpass filter in conjunction with highly chromatically corrected imaging objectives can yield subcellular resolution for imaging of the interior refractive index of human mesenchymal stem cells.
\end{abstract}

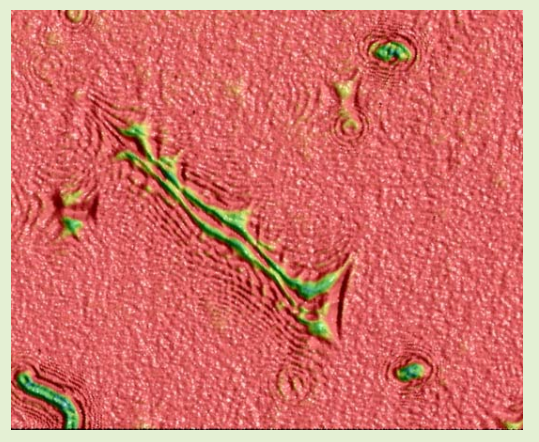

Index Terms-Biomarker, greyscale image, liquid crystal filter, mesenchymal stem cells.

\section{INTRODUCTION}

$\mathbf{H}$ UMAN mesenchymal stem cells (hMSCs) are nonhaematopoietic multipotent cells with the ability to differentiate into three lineage types such as ectoderm (neurocytes), mesoderm (osteocytes, adipocytes and chondrocyte) and endoderm (hepatocytes). The employment of hMSCs in treatment of chronic diseases like neurodegenerative diseases, autoimmune and cardiovascular diseases requires careful preclinical and clinical examinations [1]. Over the last four decades, many experimental techniques have been developed for differentiation of the stem cells towards osteogenesis involving cell interaction within the cell community [2]. It is now possible to use MSCs site- specific delivery vehicles to repair cartilage, bone, tendon, marrow stroma, muscle, and other connective tissues. For example, using the fusion protein of tissue-specific (e.g., muscle or bone

Manuscript received May 4, 2020; revised May 19, 2020; accepted May 19, 2020. Date of publication June 3, 2020; date of current version September 3, 2020. This work was supported by the Engineering and Physical Sciences Research Council, U.K., under Grant DT/E010857/1. The associate editor coordinating the review of this article and approving it for publication was Prof. Kazuaki Sawada. (Corresponding author: Asim K. Ray.)

Thomas Wilkop is with the Department of Physiology, University of Kentucky, Lexington, KY 40506 USA (e-mail: twi272@g.uky.edu).

N. Manivannan and W. Balachandran are with the Department of Electronic and Computer Engineering, Brunel University London, Uxbridge UB8 3PH, U.K. (e-mail: nadarajah.manivannan @ brunel.ac.uk; wamadeva.balachandran@brunel.ac.uk).

Asim K. Ray was with the School of Engineering and Materials Science, Queen Mary University of London, London E1 4NS, U.K. He is now with Brunel University London, Uxbridge UB8 3PH, U.K. (e-mail: asim.ray@brunel.ac.uk).

Digital Object Identifier 10.1109/JSEN.2020.2997742 marrow) peptides hooked to the outer surface of a cell, it is possible to paint tissue-specific targeting molecules onto MSCs or other reparative cells to uniquely address tissue [3]. Irradiation induced DNA damages during chemotherapy of cancer patients are readily repaired in hMSCs, but remain somewhat impaired through the induction of apoptosis [4]. Careful investigation, over a six months period, into the effect of intracerebral transplantation of hMSCs onto the basal ganglia region of nonhuman primates demonstrated a safe alternative for clinical application of neurological disorders [5]. The bone morphogenetic proteins are found from X-ray imaging studies to promote differentiation of human dermal-derived fibroblast cells in vivo [6]. The significant increase in nuclear magnetic resonance signal was observed for the $1.3 \mathrm{ppm}$ level of lipid methylene during the three days long adipogenic differentiation of hMSCs, indicating the use of the lipid metabolites in hMSC adipogenesis [7], [8].

In recent years, considerable efforts have been spent on the development of optical microscopic techniques for monitoring hMSCs differentiation with applications in regenerative medicine and cancer therapy [9]. Raman spectroscopy is regarded as being a non-invasive, label free technique for the differentiation of hMSCs into adipocytes and chondrocyte [10]. Confocal Raman spectra of osteogenic and nonosteogenic cultured hMSCs show the formation of a bone-like apatite mineral during the differentiation of hMSCs. towards an osteogenic line [11]. Raman spectra of silver nanoparticles treated hMSCs show the increase in the intensity of the methionine-related peak at $700 \mathrm{~cm}^{-1}$ due to oxidative stress for nanoparticles concentration greater than $2 \mu \mathrm{g} / \mathrm{ml}$ [12]. 


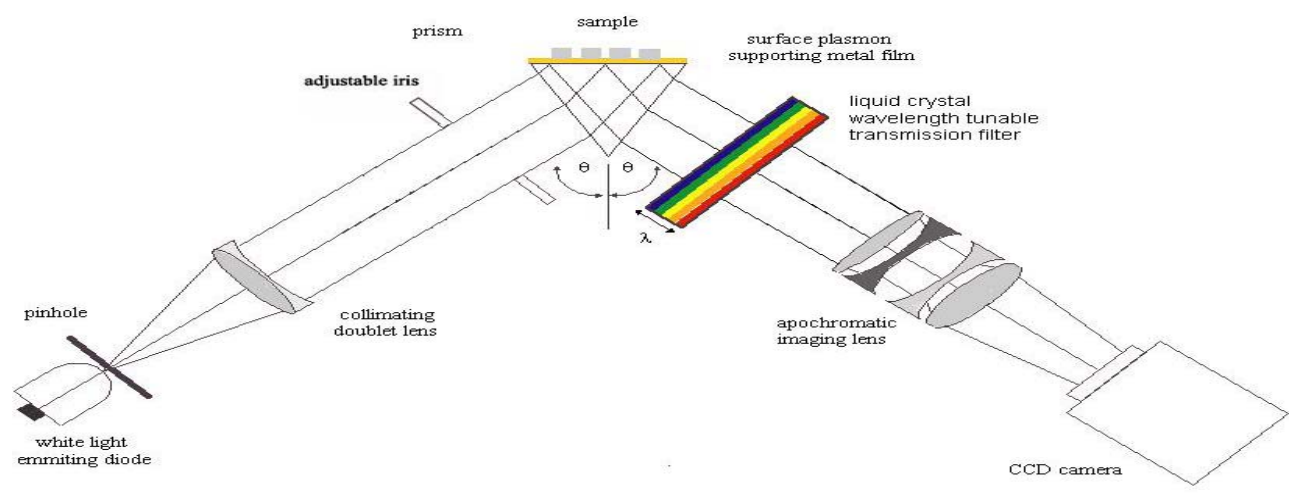

Fig. 1. Schematic of the SPR imager.

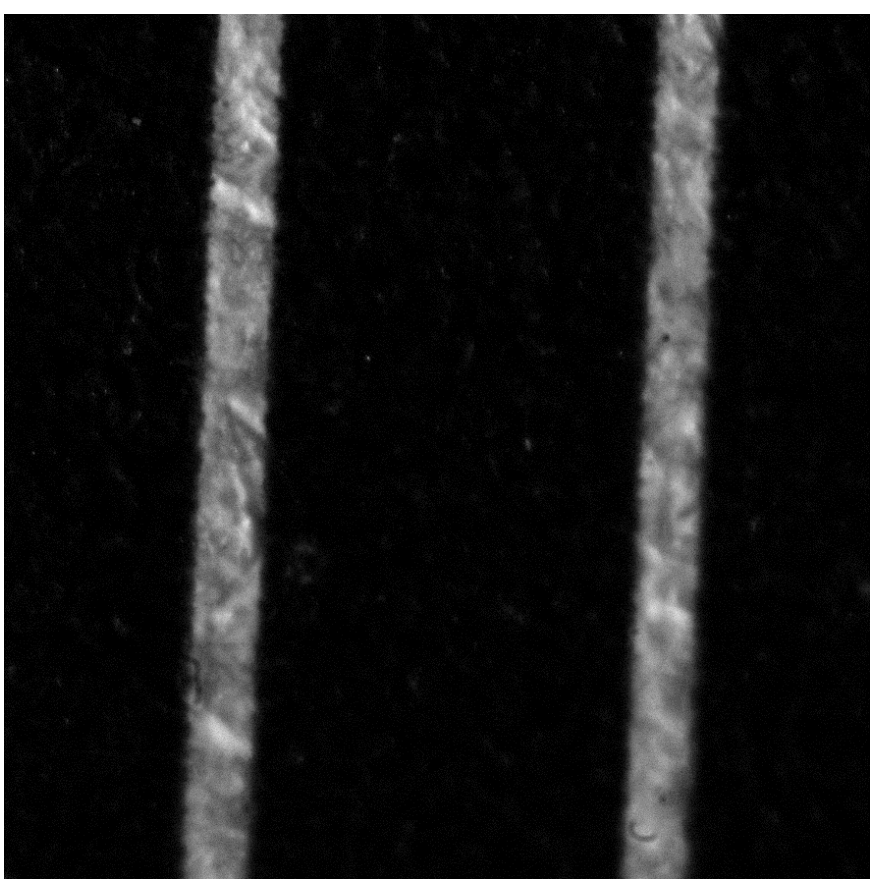

Fig. 2. Large field of view, maximum zoomed out.

Focal plane array-Fourier transform infrared spectroscopy is found to monitor rapid and sensitive chondrogenics such as collagenic and proteoglycanic differentiation of chondrocytes derived from hMSCs [13]. The role of cadmium sulfide (CdS) quantum dots in biomarking hMSCs has been studied by the photoluminescence spectroscopy for $\mathrm{CdS}$ nanoparticles of 2.4-2.8 $\mathrm{nm}$ in diameters functionalised with chitosan-Ophospho-L-serine conjugates. The fluorescent positive population becomes nearly two times larger for functionalised cells than untreated ones. The distribution of $\mathrm{CdS}$ is observed from the TEM images throughout the cytoplasm via endocytosis pathways [14].

Gold nanoparticles of varying size, with characteristic SPR coupling and hence absorption spectra, as long term tracers

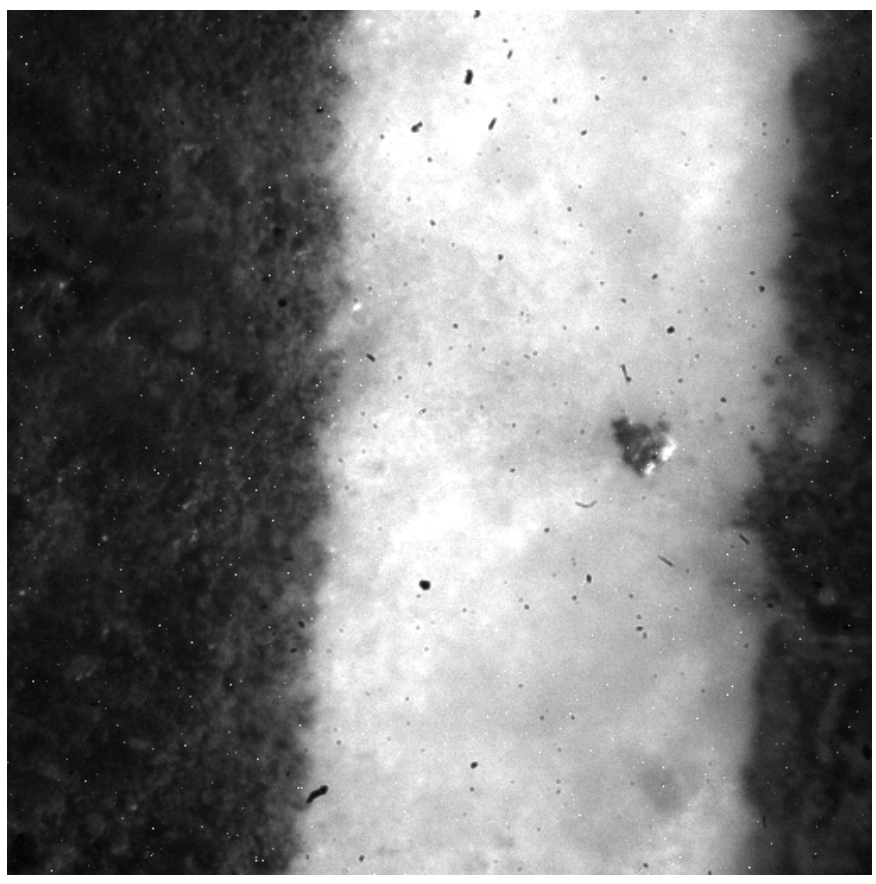

Fig. 3. Maximum zoom in, smallest field of view. (The observable "hotspots" are an artifact caused by the long exposure time).

for cell activities have shown that hMSCs differentiation is dependent upon the nanoparticle size [15]. Using the vascular endothelial-cadherin (VE) protein as a biomarker, SPR has been successfully employed to monitor the hMSC differentiation into endothelial lineage over fourteen days with the detectability of 27 cells $/ \mathrm{mm}^{2}$. In-situ increase of the SPR signal with the VE on the cell surface during the differentiation indicates the possibility of real-time live cell diagnostic treatment without any need for cell breakage [16]. In this article we report the 2-dimensional interrogation of cultured human bone marrow samples immobilized on SPR supporting substrates with a recently constructed SPR instrument that eliminates mechanically moving parts. The real-time identification of mesenchymal stem cells has proven to be both problematic and 


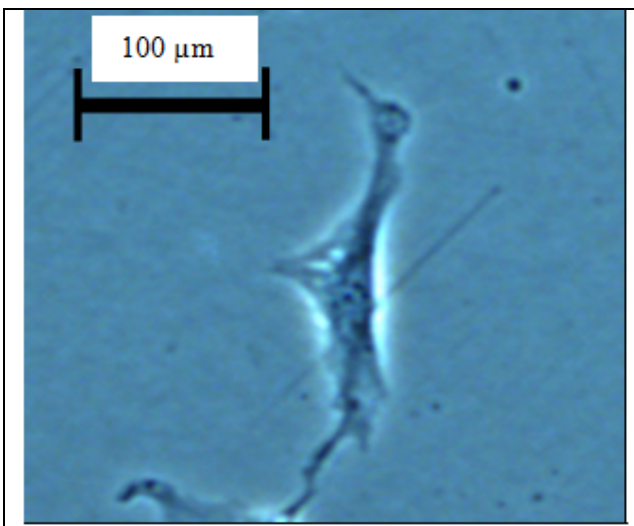

(a)

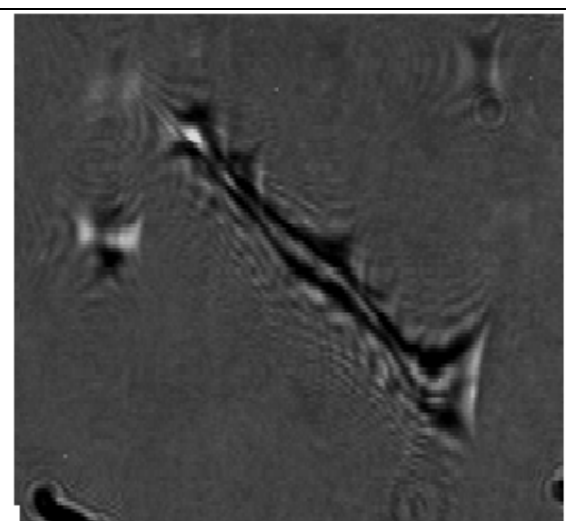

(b)

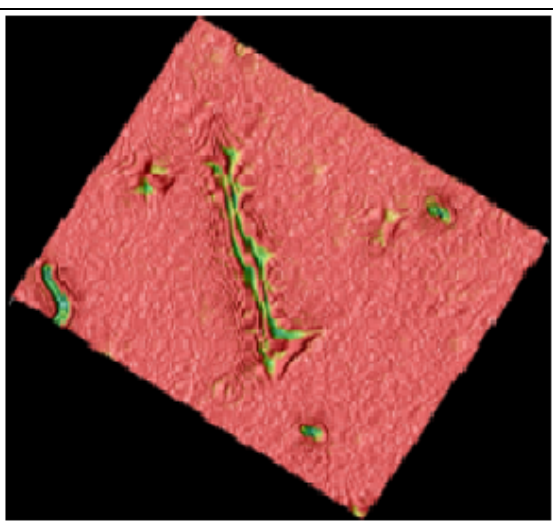

(c)

Fig. 4. Comparison of images of light microscopy against SPR, Image obtained from a light microscope (far left) at $100 \times$ magnification against the images obtained from the SPR (a) light microscope image, (b) 2D SPR image and (c) false color image.

remains elusive. Surface plasmon resonance (SPR), a highly surface-sensitive technique, may be used for the real time high throughput examination of specific cell types based on their dielectic/refractive index properties [17]. By using a liquid crystal Fabry Perot wavelength tunable transmission filter the instrument features a small footprint and a high resolution axial resolution. A schematic of wavelength spectroscopic SPR microscope/imager is shown in Figure1. Depending upon the relaxation time of the liquid crystal, the scan speed can exceed that of a goniometer based angular rotation SPR system [18].

\section{EXPERIMENTAL DETAILS}

Human mesenchymal stem cells (hMSCs) were donated by the life sciences company, Progentix BV (The Netherlands). These cells were chosen owing to their ability to attach to a wide variety of surfaces, including those composed of gold and titanium. The bone marrow was placed in T-flasks, expanded in culture medium, harvested and cryopreserved. The cells were cultured on SPR supporting substrates ( $\mathrm{Cr}$ seedlayer plus $\mathrm{Au}$ ) covered with an ultrathin layer of fetal bovine serum [19]. Further information is available in our earlier publication [20]. In order to facilitate imaging of the cells in air a standard protocol was used to fixate the cells with formaldehyde. The established preservation of cells with formaldehyde allow it to retain fine cellular structures.

A schematic of the spectroscopic SPR microscope is shown in Figure1. The output of a super bright white 5 Watt LED, operated in stabilized constant current mode, is passed through a pinhole (ca.100 $\mu \mathrm{m}$ ) and collimated with a doublet lens $\mathrm{f}$ $=160 \mathrm{~mm}$ to a beam diameter of $25 \mathrm{~mm}$. An adjustable iris reduces the beam diameter down to the sample seize. The advantage of using incoherent light in SPR microscopy is the elimination of coherence artefacts and speckle found in laser based systems. Otherwise these require either rotating diffuser or spatial filter to improve the image quality. The InGaN LED uses a single emitting die with a phosphor coating to generate white light. The emission spectra show the characteristic white LED profile with a strong peak in the blue and a hump in the green-red (SP 1). The illumination optics is mounted on a manual goniometer arm of an manual dispersion spectrometer to allow selection of the angle of incidence. Measurements in air employ equilateral BK7 prisms, measurements in aqueous media equilateral SF2 prisms. One face of the prism is coated with a SPR supporting metal, either ca. $47 \mathrm{~nm}$ of gold or ca. $50 \mathrm{~nm}$ of silver. The totally reflected light beam passes through a Varispec filter (Cambridge Research Instrumentation), an electronically tunable liquid crystal filter (LCTF) that allows computer controlled selection of the transmission spectrum. The filter is polarization sensitive and orientated to passes only p-polarized light that excites surface plasmons. The Varispec filter is based on the Lyot filter principle; it is constructed from a stack of polarizer and electronically tunable retardation (birefringent) liquid crystal plates. The transmission window of the filter is Gaussian throughout, with a variable transmission peak height and a transmission bandwidth that increases slightly toward the red end of the spectra. The reflected light from the sample, which has passed through the filter, is recorded with a 2 megapixel CCD camera mounted onto a highly apochromatic $65 \mathrm{~mm}$ multi element lens that is synchronized with the wavelength selection of the filter. The great benefit of wavelength interrogation of the SPR reflectivity response is the elimination of angular distortions that are a standard feature of conventional theta 2 theta rotation scans. Wavelength interrogation provides pixel accuracy resolution for the measurements of small samples that retain their exact dimension.

\section{Results}

Depending on the refractive index distribution in the cell adhering to the substrate surface varying SPR coupling efficiencies can be observed across the cell [21]. Figure 2 is recorded at the maximum optical resolution that is obtainable by the constructed instrument for an imaging distance of $25 \mathrm{~mm}$, i.e. the distance that separates the imaging optics from the back for the object using the Kretschman configuration. Using custom made smaller prism could increase the lateral resolution or conversely increasing the imaging distance can enlarge the field of view. The images show a segment from a resolution chart (Edmund Optics, Barrington, New Jersey). The white stripe depicted has a width of approximate $0.6 \mathrm{~mm}$ 

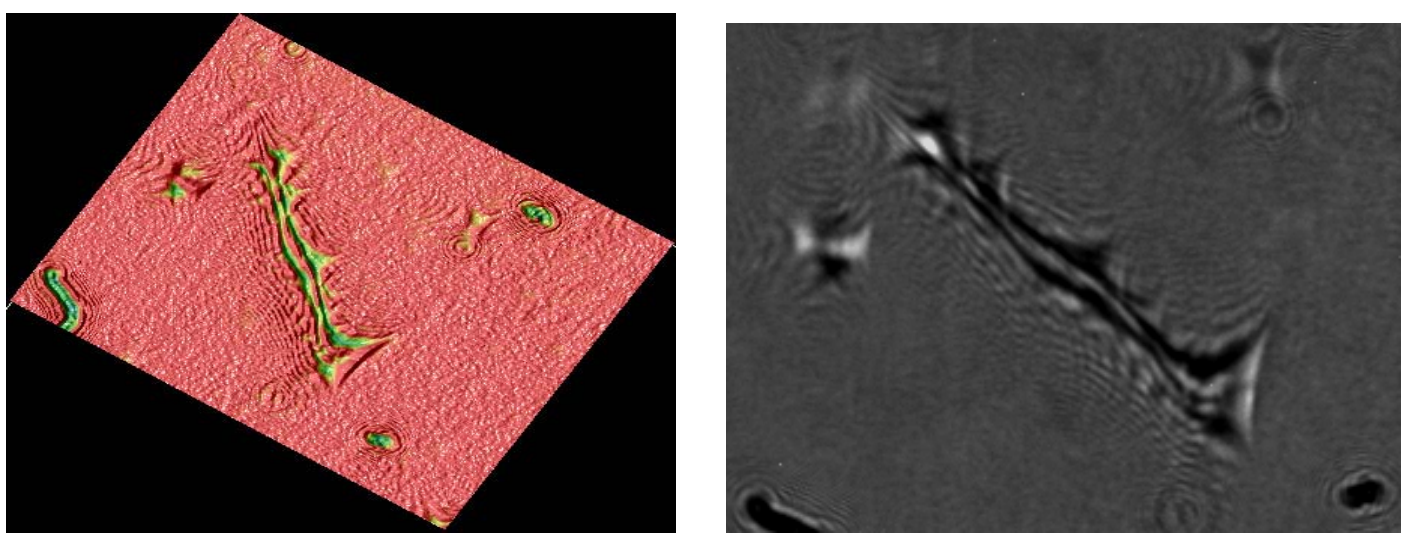

(a)
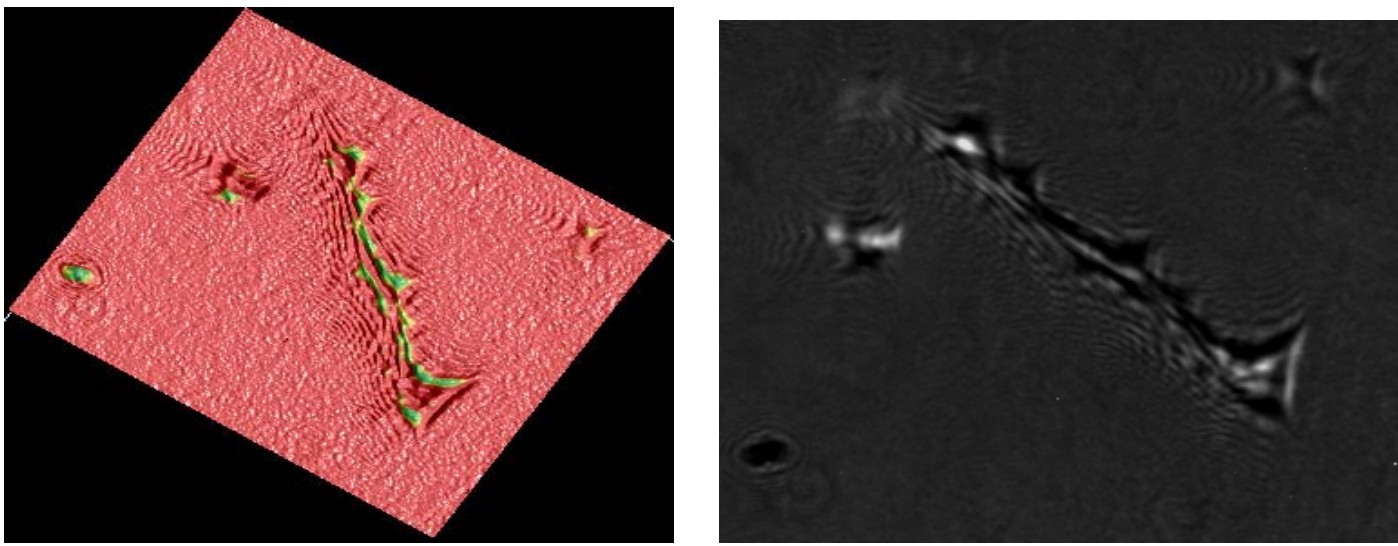

(b)
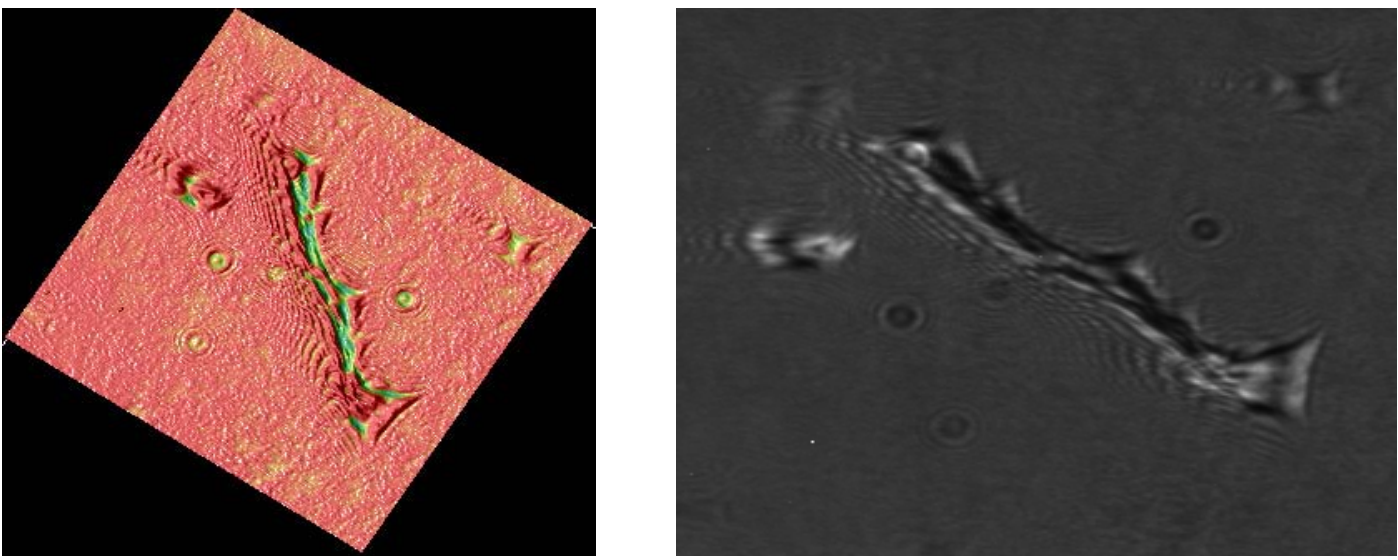

(c)

Fig. 5. External angle of incidence (a) $25^{\circ}$ (b) $29^{\circ}$ and (c) $31.5^{\circ}$ at $560 \mathrm{~nm}$ center wavelength.

TABLE I

CONTRAst of IMAGES With Standard DEVIATION

\begin{tabular}{|c|c|c|}
\hline Angle of incidence & Contrast & Standard deviation of the background \\
\hline $25^{0}$ & 243 & 11.0 \\
\hline $29^{0}$ & 250 & 5.0 \\
\hline $31^{0}$ & 245 & 2.3 \\
\hline
\end{tabular}

and the pitch between adjacent stripes is $4 \mathrm{~mm}$. The magnification is user selectable by adjusting the lens position and zoom to investigate samples of widely different dimensions. The instrument resolution is suitable for morphological studies of chitosanO-phospho-L-serine conjugates functionalised with fluorescent cadmium sulfide quantum dots [14] as biomarkers when imaging and detecting bone tissue regeneration and metabolic events. 


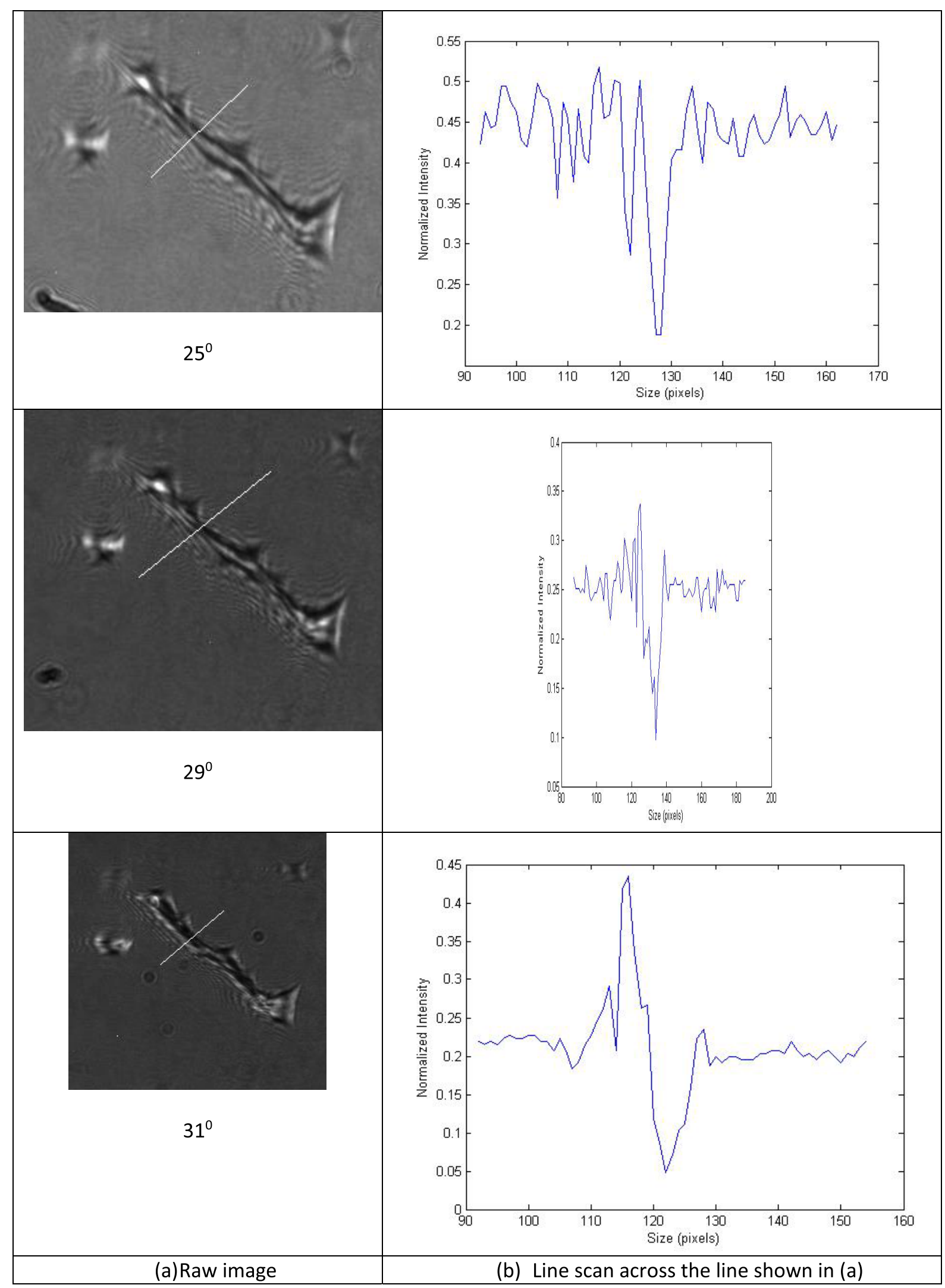

Fig. 6. Intensity analysis of raw images in Figure 5.

The observed "hotspots" in Figure 3 are an artifact of the long exposure time and state of the CCD. Different parts of the cell appear brighter depending on the local refractive index affecting (minimizing) the coupling of the wave vectors on the incoming light into surface plasmons, underscoring the lable free contrast mechanism. Artifacts in conventional radiographic images which depend upon the image plates, plate readers, image processing software are difficult to 
identify [22]. This problem does not exist in the present investigation.

As shown in Figure 4, structural assessments can be made from all three images. Fibroblastic morphology is readily defined despite the different means of obtaining the images. The light microscope image demonstrates a 2D colour image in Figure 4(a) with high clarity and resolution. The 2D SPR image in Figure 4(b) possesses modest clarity and resolution. Additional information may be obtained through the ripple effects noted around the cell, where the surface plasmon decay length and onset might contribute. The image in Figure 4(c) is a false color rendering of the cell, in which the green part indicate less effective SPR coupling over the red part.

Figure 5 shows selected images from a scan of immobilized cells. Images are presented in both their raw format, and to aid visualization in a false colour plot. Depending on the refractive index distribution in the cell, different coupling SPR coupling efficiencies are clearly observable, i.e. the interior of the cells cytoplasm and organelle structures are imaged based on their refractive index/ dielectric constant. Given that the cells are non absorbing, at the used wavelength, we are confident that contrast is due to variations in the real part of the refractive index $\mathrm{n}$. Adjusting the angle highlights subtle variations in the cells interior refractive index fairly dramatically. SPR imaging/microscopy is able to examine the bacterial cells at the subcellular resolution level and with finely nuanced refractive index resolution [24].

Images were processed within MATLAB. The colour images was first converted into greyscale images using the MATLAB built-in function (known as RGB2GRAY). The algorithm used for this conversion are given as below, where $R, G$ and $B$ are the intensity values of Red, Green and Blue respectively and I represents the intensity of the greyscale image in the form [25].

$$
I=0.2989 R+0.5870 G+0.1140 B
$$

Figure 6 shows (a) raw image with a line, (b) line scan of intensity along the line in the image (a) using Eq (1). The line scans provide a detailed assessment of the strong obtainable image contrast and quality for various angle of incidence, with $31^{0}$ yielding, due to strongest coupling, highest values. The contrast of the images was calculated as the difference between the max intensity and minimum intensity and they do not show much variation between them. Values are summarised in Table I including the standard deviation of the background. As can be seen, the standard deviation is lowest for the incidence angle of 31 degree and highest for 25 degree which is in accordance with results shown in Figure 6(b).

\section{COncluding Remarks}

The real-time interrogation of mesenchymal stem cells has proven to be both problematic and remains elusive. A surface plasmon resonance (SPR) microscope/imager that generates articulation free, a continuous reflectivity spectrum versus wavelength, has been developed. The instrument allows the measurements of the wavelength dependent surface plasmon resonance reflectivity spectrum of a 2 dimensional sample across the entire visible spectrum without any mechanically moving parts. Further, the compact simple design from standard components and low power consumption for the examination of sub cellular details is very attractive. With our unique lateral distortion free imaging principle, which maintains pixel accuracy throughout the measurement, we were able to demonstrate a scan of cellular features of human mesenchymal stem cells. This paper provides proof of principle that a variable transmission filter based SPR microscope can be indeed used as a platform for studying cellular phenomena. The real-time identification of mesenchymal stem cells has proven to be both problematic and elusive for over four decades. SPR, a highly surface-sensitive technique, may be used to obtain distinct spectra that are revealing intracellular compositional changes affecting the local refractive index toward identification of specific cell types. Any such real-time identification would prove cost effective and time-saving, especially within the downstream processes of cell production industries.

\section{ACKNOWLEDGMENT}

The authors are grateful for Dr. A. S. Ramlogan, and Prof. J. D. de Bruijn for the sample preparation.

\section{ReFERENCES}

[1] I. Ullah, R. B. Subbarao, and G. J. Rho, "Human mesenchymal stem cells-current trends and future prospective," Biosci. Rep., vol. 35 , Apr. 2015, Art. no. e00191, doi: 10.1042/BSR20150025.

[2] A. J. Friedenstein, S. Piatetzky-Shapiro, and K. V. Petrakova, "Osteogenesis in transplants of bone marrow cells," Development, vol. 16, no. 3, pp. 381-390, 1966.

[3] A. I. Caplan, "Mesenchymal stem cells: Cell-based reconstructive therapy in orthopedics," Tissue Eng., vol. 11, nos. 7-8, pp. 1198-1211, 2005.

[4] L. Oliver et al., "Distinct roles of BCL-2 and BCL-XL in the apoptosis of human bone marrow mesenchymal stem cells during differentiation," PLoS ONE, vol. 6, no. 5, May 2011, Art. no. e19820, doi: 10.1371/journal.pone.0019820.

[5] M. Feng et al., "Preclinical safety evaluation of human mesenchymal stem cell transplantation in cerebrum of nonhuman primates," Int. J. Toxicol., vol. 33, no. 5, pp. 403-411, Sep. 2014, doi: $10.1177 / 1091581814545244$.

[6] F. Chen et al., "Bone morphogenetic protein 7-transduced human dermal-derived fibroblast cells differentiate into osteoblasts and form bone in vivo," Connective Tissue Res., vol. 59, no. 3, pp. 223-232, 2018, doi: 10.1080/03008207.2017.1353085.

[7] S.-I. Chun, J.-H. Cho, Y. I. Yang, J.-W. Shin, W.-J. Shin, and C.-W. Mun, "Proton $(1 \mathrm{H})$ nuclear magnetic resonance spectroscopy to define metabolomic changes as a biomarker of adipogenic differentiation in human mesenchymal stem cells," Tissue Eng. Regenerative Med., vol. 9, no. 2, pp. 101-108, Apr. 2012, doi: 10.1007/s13770-012-0016-6.

[8] A. Downes, R. Mouras, and A. Elfick, "Optical spectroscopy for noninvasive monitoring of stem cell differentiation," J. Biomed. Biotechnol., vol. 2010, pp. 1-10, 2010, doi: 10.1155/2010/101864.

[9] M. Pudlas, S. Koch, C. Bolwien, T. Hirth, H. Walles, and K. Schenke-Layland, "Raman spectroscopy: A powerful tool for the non-contact discrimination of bone marrow mesenchymal stem cells and fibroblasts," Proc SPIE, vol. 7903, Feb. 2011, Art. no. 79032I, doi: 10.1117/12.873047.

[10] L. L. McManus, G. A. Burke, M. M. McCafferty, P. O’Hare, M. Modreanu, A. R. Boyd, and B. J. Meenan, "Raman spectroscopic monitoring of the osteogenic differentiation of human mesenchymal stem cells," Analyst, vol. 136, no. 12, pp. 2471-2481, 2011, doi: 10.1039/c1an15167c.

[11] A. Bankapur et al., "Micro-Raman spectroscopy of silver nanoparticle induced stress on optically-trapped stem cells," PLOS ONE, vol. 7, no. 4, Apr. 2012, Art. no. e35075, doi: 10.1371/journal.pone.0035075.

[12] C. Chonanant et al., "Discrimination of micromass-induced chondrocytes from human mesenchymal stem cells by focal plane array-Fourier transform infrared microspectroscopy," Talanta, vol. 130, pp. 39-48, Dec. 2014, doi: 10.1016/j.talanta.2014.05.037. 
[13] S. Ristig, S. Chernousova, W. Meyer-Zaika, and M. Epple, "Synthesis, characterization and in vitro effects of $7 \mathrm{~nm}$ alloyed silver-gold nanoparticles," Beilstein J. Nanotechnol., vol. 6, pp. 1212-1220, May 2015, doi: 10.3762/bjnano.6.124.

[14] C. L. Salgado, A. A. P. Mansur, H. S. Mansur, and F. J. M. Monteiro, "Fluorescent bionanoprobes based on quantum dot-chitosan-O-phospho1-serine conjugates for labeling human bone marrow stromal cells," RSC $A d v$, vol. 4, no. 90, pp. 49016-49027, 2014, doi: 10.1039/c4ra08247h.

[15] J.-H. Fan, W.-T. Li, W.-I. Hung, C.-P. Chen, and J.-M. Yeh, "Cytotoxicity and differentiation effects of gold nanoparticles to human bone marrow mesenchymal stem cells," Biomed. Eng., Appl., Basis Commun., vol. 23 , no. 2, pp. 141-152, Apr. 2011, doi: 10.4015/S1016237211002475.

[16] F. Fathi, A. Rezabakhsh, R. Rahbarghazi, and M.-R. Rashidi, "Early-stage detection of VE-cadherin during endothelial differentiation of human mesenchymal stem cells using SPR biosensor," Biosensors Bioelectron., vol. 96, pp. 358-366, Oct. 2017, doi: 10.1016/j.bios.2017.05.018.

[17] S. Paul, P. Vadgama, and A. K. Ray, "Surface plasmon resonance imaging for biosensing," IET Nanobiotechnology, vol. 3, no. 3, p. 71, 2009, doi: 10.1049/iet-nbt.2008.0012.

[18] H. J. Zhang, J. H. Dai, and P. Y. Wang, "Measurement of relaxation time of liquid crystal by bifurcation in optical bistability," in Laser Spectroscopy VII (Springer Series in Optical Sciences), vol. 49, T. W. Hänsch and Y. R. Shen, Eds. Berlin, Germany: Springer, 1985.
[19] S. C. Mendes, I. Van Den Brink, J. D. De Bruijn, and C. A. Van Blitterswijk, "In vivo bone formation by human bone marrow cells: Effect of osteogenic culture supplements and cell densities,' J. Mater. Sci., Mater. Med., vol. 9, no. 12, pp. 855-858, Dec. 1998.

[20] T. Wilkop, A. S. Ramlogan, I. L. Alberts, J. D. de Bruijn, and A. K. Ray, "Surface plasmon resonance imaging for medical and biosensing," in Proc. IEEE Sensors, Oct. 2009, pp. 1571-1574, doi: 10.1109/ICSENS.2009.5398485.

[21] L. Sun et al., "Refractive index sensing and imaging based on polarization-sensitive graphene," Opt. Express, vol. 27, no. 20, p. 29273 , Sep. 2019, doi: 10.1364/OE.27.029273.

[22] L. J. Cesar, B. A. Schueler, F. E. Zink, T. R. Daly, J. P. Taubel, and L. L. Jorgenson, "Artefacts found in computed radiography," Brit. J. Radiol., vol. 74, no. 878, pp. 195-202, Feb. 2001.

[23] T. Kawakami and S. J. Galli, "Regulation of mast-cell and basophil function and survival by IgE," Nature Rev. Immunol., vol. 2, no. 10, pp. 773-786, Oct. 2002, doi: 10.1038/nri914.

[24] T.-F. Ma, Y.-P. Chen, J.-S. Guo, W. Wang, and F. Fang, "Cellular analysis and detection using surface plasmon resonance imaging," TrAC Trends Anal. Chem., vol. 103, pp. 102-109, Jun. 2018.

[25] Q. Liu, J. Xiong, L. Zhu, M. Zhang, and Y. Wang, "Extended RGB2Gray conversion model for efficient contrast preserving decolorization," Multimedia Tools Appl., vol. 76, no. 12, pp. 14055-14074, Jun. 2017. 